\title{
A Goodness of Fit Test For Normality Based on Balakrishnan- Sanghvi Information
}

\author{
Mahsa Tavakoli ${ }^{1}$, Naserreza Arghami ${ }^{2}$, and Malihe Abbasnejad ${ }^{3}$. \\ ${ }^{1}$ Department of Statistics, Ferdowsi University of Mashhad, Iran. \\ ${ }^{2}$ Department of Statistics, Ferdowsi University of Mashhad, Iran. \\ ${ }^{3}$ Department of Statistics, Hakim Sabzevari University of Mashhad, Iran.
}

Received: 12/07/2017, Revision received: 04/06/2018, Published online: 03/28/2019

\begin{abstract}
We introduce a new goodness of fit test for normality based on BalakrishnanSanghvi divergence measure. In order to estimate the divergence measure, we use a method similar to Vasicek's for estimating the Shannon entropy. Also, the test statistic based on kernel density estimation is investigated. Critical values and the power of tests are computed by Monte Carlo simulation. It is shown that the tests are consistent. Further, by comparing the power of proposed tests with other normality tests, we suggest the new entropic based test according to Balakrishnan-Sanghvi divergence measure using Vasicek method.
\end{abstract}

Keywords. Divergence measure, Entropy, Normality test, Vasicek sample entropy.

MSC: 62G05; 62G10; 62G20.

Corresponding Author: Mahsa Tavakoli (mahsa_tavakoli88@yahoo.com)

Naserreza Arghami (arghami_nr@yahoo.com)

Malihe Abbasnejad (m.abbasnejad@hsu.ac.ir) 


\section{Introduction}

The goodness of fit test for normality has been widely discussed in different literature. Normal distribution plays a predominant role in various sciences like management, engineering and reliability. So in many applications, we need to test whether the underlying data follow a normal distribution or not. Since the introduction of the chisquared goodness of fit test for normality by Pearson in 1900, considerable attention has been given to the problem of testing normality and various tests, such as the moment-based test, regression-based test and entropy-based test.

Let $f$ denote the density of a given population, then the null and alternative hypotheses of normality are stated formally as $H_{0}: f(x)=f_{0}(x ; \mu, \sigma)$ and $H_{1}: f(x) \neq f_{0}(x ; \mu, \sigma)$, where $\mu$ and $\sigma$ are unknown parameters such that

$$
f_{0}(x ; \mu, \sigma)=\frac{1}{\sqrt{2 \pi} \sigma} \exp \left\{-\frac{1}{2}\left(\frac{x-\mu}{\sigma}\right)^{2}\right\} .
$$

Cramer Von Mises and Kolmogorov-Smirnov proposed their tests in 1931 and 1933 respectively. Some other popular tests for normality founded by Shapiro-Wilk (1965), Anderson-Darling (1954), Kuiper (1960) and Watson (1961). For the first time, Vasicek (1976) introduced a normality test based on entropy. The entropy of $X$ with distribution function $F(x)$ and a continuous density function $f(x)$ is defined by Shannon (1948) as

$$
H(f)=-\int_{-\infty}^{\infty} f(x) \log f(x) d x
$$

The problem of estimating Shannon entropy has been considered by many authors, including Ahmad and Lin (1976), Vasicek (1976), Dudewicz and Van der Meulen (1981), Joe (1989), Van Es (1992), Ebrahimi et al. (1994), Corea (1995), Wieczorkowski and Grzegorzewski (1999), Yousefzadeh and Arghami (2008) and Alizadeh (2010). Among these various entropy estimators, Vasicek's sample entropy has been the most widely used in developing entropy-based statistical procedures. With the transformation $F(x)=p$, Vasicek (1976) rewrote equation (1) as

$$
H(f)=\int_{0}^{1} \log \left\{\frac{d}{d p} F^{-1}(p)\right\} d p .
$$

By replacing the distribution function $F$ with its empirical distribution function $F_{n}$ and using the well-known difference operator instead of the used differential operator, the 
estimator of $H(f)$ is given as

$$
H_{n, m}=\frac{1}{n} \sum_{i=1}^{n} \log \left\{\frac{n}{2 m}\left(X_{(i+m)}-X_{(i-m)}\right)\right\},
$$

where $X_{(1)} \leq \cdots \leq X_{(n)}$ are the order statistics and $m$ is a positive integer, $m \leq n / 2$ and $X_{(i)}=X_{(1)}$ if $i<1, X_{(i)}=X_{(n)}$ if $i>n$.

The sample entropy was considered in establishing a goodness of fit test statistic for normal distribution by Vasicek (1976) and Arizono and Ohta (1989), also Dudewicz and Van der Meulen (1981) used it for uniform distribution, and Ebrahimi et al. (1992) applied it for the exponential distribution.

Vasicek (1976) introduced the test statistic based on the property of maximum entropy of the normal distribution as

$$
K_{n, m}=\frac{\exp \left(H_{n, m}\right)}{\hat{\sigma}}=\frac{n}{2 m \hat{\sigma}}\left(\prod_{i=1}^{n}\left[X_{(i+m)}-X_{(i-m)}\right]\right)^{1 / n}
$$

where

$$
\hat{\sigma}=\sqrt{\frac{1}{n} \sum_{i=1}^{n}\left(X_{i}-\bar{X}\right)^{2}}
$$

Park (1999) provided a test of normality based on the sample entropy of order statistics. Choi (2008) improved Vasicek's entropy test as

$$
K_{n, m}^{c}=\frac{R_{n, m}}{\hat{\sigma}_{n-1}}\left(\prod_{i=1}^{n}\left[X_{(i+m)}-X_{(i-m)}\right]\right)^{1 / n},
$$

where

$$
R_{n, m}=\exp \left\{-(1-2 m / n) \psi(2 m)+\psi(n+1)-2 / n \sum_{i=1}^{n} \psi(k+m-1)\right\},
$$

and $\psi$ is the digamma function such that

$$
\psi(t)=\frac{d \ln \Gamma(t)}{d t}=\frac{d \ln \int_{0}^{\infty} x^{t-1} t^{x} d x}{d t}
$$


and $\hat{\sigma}_{n-1}=\{n /(n-1)\}^{1 / 2} \hat{\sigma}$.

Esteban et al. (2001) compared four tests of normality using four statistics based on different entropy estimators namely, Vasicek (1976), Van Es (1992), Corea (1995) and Wieczorkowski and Grzegorzewski (1999). Similar to our work, Alizadeh (2010) compared the four mentioned tests with a test based on a new estimator of entropy as

$$
T A_{m n}=\frac{\exp \left\{H_{a}(m, n)\right\}}{\hat{\sigma}}
$$

where $\hat{\sigma}=\sqrt{\frac{1}{n} \sum_{i=1}^{n}\left(X_{i}-\bar{X}\right)^{2}}$ and $H_{a}(m, n)$ is the new estimator of entropy as

$$
H_{a}(m, n)=-\frac{1}{n} \sum_{i=1}^{n} \log \left\{\frac{\hat{f}\left(X_{(i+m)}\right)+\hat{f}\left(X_{(i+m)}\right)}{2}\right\},
$$

where $\hat{f}(X)=\frac{1}{n h} \sum_{i=1}^{n} k\left(\frac{X-X_{(i)}}{h}\right)$, and the kernel function $k$ is chosen to be the standard normal density function and the bandwidth $h$ is chosen to be the normal optimal smoothing formula with $h=1.06 s n^{-\frac{1}{5}}$ where $s$ is the sample standard deviation.

In other works, Alizadeh and Arghami (2011a) compared seven different tests of normality such as the Kolmogorov-Smirnov (1933), Anderson-Darling (1954), Kuiper (1960), Jarque-Bera (1987), Cramer-Von Mises (1931), Shapiro-Wilk (1965) and Vasicek (1976).

This paper is organized as follows. In Section 2, we introduce two test statistics based on Balakrishnan-Sanghvi divergence measure. These statistics are estimated in the manner of Vasicek for estimating the entropy and also estimated using kernel density function. Other methods of testing normality like Corea (1995) and Van Es (1992) are investigated, but their results are not given because of their weak performances. Also in this section, we show the tests are asymptotically consistent. In Section 3, using Monte Carlo simulation, it is shown that the proposed test based on Vasicek method has greater power than its competitors in most of the times. 


\section{Test statistics}

Balakrishnan-Sanghvi divergence measure (Balakrishnan and Sanghvi (1968) ) for two density function $f(x)$ and $g(x)$ is defined as

$$
D_{B S}(f, g)=\int_{-\infty}^{\infty}\left(\frac{f(x)-g(x)}{f(x)+g(x)}\right)^{2} f(x) d x .
$$

It is obvious that $D_{B S}(f, g) \geq 0$ and the equality holds if and only if $f(x)=g(x)$. So it motivates us to use Balakrishnan-Sanghvi information as a test statistic for normality. Plugging

$$
g(x)=f_{0}(x ; \mu, \sigma)=\left(1 / \sqrt{2 \pi \sigma^{2}}\right) \exp \left\{-(x-\mu)^{2} / 2 \sigma^{2}\right\}
$$

in (7), we get

$$
D_{B S}(f, g)=\int_{-\infty}^{\infty} f(x)\left(\frac{f(x)-\left(1 / \sqrt{2 \pi \sigma^{2}}\right) \exp \left\{-(x-\mu)^{2} / 2 \sigma^{2}\right\}}{f(x)+\left(1 / \sqrt{2 \pi \sigma^{2}}\right) \exp \left\{-(x-\mu)^{2} / 2 \sigma^{2}\right\}}\right)^{2} d x .
$$

Now, similar to Vasicek's method for estimating entropy, using $F(x)=p$, equation (8) is expressed as

$$
D_{B S}(f, g)=\int_{0}^{1}\left(\frac{\left(\frac{d F^{-1}(p)}{d p}\right)^{-1}-\left(1 / \sqrt{2 \pi \sigma^{2}}\right) \exp \left\{-\left(F^{-1}(p)-\mu\right)^{2} / 2 \sigma^{2}\right\}}{\left(\frac{d F^{-1}(p)}{d p}\right)^{-1}+\left(1 / \sqrt{2 \pi \sigma^{2}}\right) \exp \left\{-\left(F^{-1}(p)-\mu\right)^{2} / 2 \sigma^{2}\right\}}\right)^{2} d p .
$$

By replacing $F$ by $F_{n}$ and using the well-known the difference operator in place of differential operator, we get an estimator $B_{V}$ of $D_{B S}(f, g)$ as follow

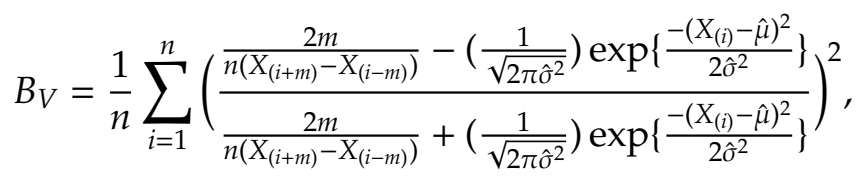

where $X_{(i)}=X_{(1)}$ for $i<1, X_{(i)}=X_{(1)}$ for $i>n$. Also $\hat{\mu}=\bar{X}$ and $\hat{\sigma}^{2}=\frac{1}{n} \sum_{i=1}^{n}\left(X_{i}-\bar{X}\right)^{2}$ are the MLEs of $\mu$ and $\sigma^{2}$ under the null hypothesis.

It is obvious that $B_{V}$ is invariant with respect to scale and location transformations. For the consistency of the test, similar to the proof of Alizadeh (2010), we prove $B_{V}$ is 
consistent. That is the power of the test under the alternative hypothesis increases to one as $n \rightarrow \infty$. The following statement shows this.

Theorem 2.1. Let $F$ be an unknown continuous distribution on the real line and let $F_{0}$ be distributed as a normal distribution with unknown parameters. Then the test based on $B_{V}$ is consistent.

Proof. As $n, m \rightarrow \infty$ and $m / n \rightarrow 0$, we have

$$
\begin{aligned}
\frac{2 m}{n} & =F_{n}\left(X_{(i+m)}\right)-F_{n}\left(X_{(i-m)}\right) \simeq F\left(X_{(i+m)}\right)-F\left(X_{(i-m)}\right) \\
& \simeq \frac{f\left(X_{(i+m)}\right)+f\left(X_{(i-m)}\right)}{2}\left(X_{(i+m)}-X_{(i-m)}\right),
\end{aligned}
$$

where $F_{n}(a)=\#\left(x_{i} \leq a\right) / n=(1 / n) \sum I_{\left(-\infty, X_{i}\right]}(a)$ and $I$ is the indicator function. Therefore noting that $\hat{\mu}$ and $\hat{\sigma}^{2}$ are consistent estimators, we have

$$
\begin{aligned}
B_{V} & =\frac{1}{n} \sum_{i=1}^{n}\left(\frac{\frac{2 m}{n\left(X_{(i+m)}-X_{(i-m)}\right)}-\left(\frac{1}{\sqrt{2 \pi \hat{\sigma}^{2}}}\right) \exp \left\{\frac{-\left(X_{(i)}-\hat{\mu}\right)^{2}}{2 \hat{\sigma}^{2}}\right\}}{\frac{2 m}{n\left(X_{(i+m)}-X_{(i-m)}\right.}+\left(\frac{1}{\sqrt{2 \pi \hat{\sigma}^{2}}}\right) \exp \left\{\frac{-\left(X_{(i)}-\hat{\mu}\right)^{2}}{2 \hat{\sigma}^{2}}\right\}}\right)^{2} \\
& \simeq \frac{1}{n} \sum_{i=1}^{n}\left(\frac{\frac{F\left(X_{(i+m)}\right)-F\left(X_{(i-m)}\right)}{X_{(i+m)}-X_{(i-m)}}-\left(\frac{1}{\sqrt{2 \pi \sigma^{2}}}\right) \exp \left\{\frac{-\left(X_{(i)}-\mu\right)^{2}}{2 \sigma^{2}}\right\}}{\frac{F\left(X_{(i+m)}\right)-F\left(X_{(i-m)}\right)}{X_{(i+m)}-X_{(i-m)}}+\left(\frac{1}{\sqrt{2 \pi \sigma^{2}}}\right) \exp \left\{\frac{-\left(X_{(i)}-\mu\right)^{2}}{2 \sigma^{2}}\right\}}\right)^{2} \\
& \longrightarrow E\left(\frac{\frac{F\left(X_{(i+m)}\right)-F\left(X_{(i-m)}\right)}{X_{(i+m)}-X_{(i-m)}}-\left(\frac{1}{\sqrt{2 \pi \sigma^{2}}}\right) \exp \left\{\frac{-(X-\mu)^{2}}{2 \sigma^{2}}\right\}}{\frac{F\left(X_{(i+m)}\right)-F\left(X_{(i-m)}\right)}{X_{(i+m)}-X_{(i-m)}}+\left(\frac{1}{\sqrt{2 \pi \sigma^{2}}}\right) \exp \left\{\frac{-(X-\mu)^{2}}{2 \sigma^{2}}\right\}}\right)^{2} .
\end{aligned}
$$

where the last limit holds by the law of large numbers. We have $X_{(i-m)}$ and $X_{(i+m)}$ belong to an interval in which $f(x)$ is positive and continuous, then there exists a value $X_{i}^{\prime} \in\left(X_{(i-m)}, X_{(i+m)}\right)$ such that

$$
\frac{F\left(X_{(i+m)}\right)-F\left(X_{(i-m)}\right)}{X_{(i+m)}-X_{(i-m)}}=f\left(X_{i}^{\prime}\right) .
$$

Therefore $B_{V} \rightarrow D_{B S}\left(f, f_{0}\right)$. So, the test based on $B_{V}$ is consistent.

We have estimated Balakrishnan-Sanghvi divergence measure based on spacing method. Also, one can estimate this divergence using methods like kernel-based approaches. 
The Balakrishnan-Sanghvi test statistic based on kernel density function is

$$
B_{K}=\frac{1}{n} \sum_{i=1}^{n}\left(\frac{\hat{f}\left(x_{i}\right)-g\left(x_{i}\right)}{\hat{f}\left(x_{i}\right)+g\left(x_{i}\right)}\right),
$$

in which $f$ can be replaced by the kernel density estimator

$$
\hat{f}\left(x_{i}\right)=\frac{1}{n h} \sum_{j=1}^{n} k\left(\frac{x_{i}-x_{j}}{h}\right),
$$

where the kernel function $k$ is the standard normal density function and the bandwidth $h$ is chosen to be the normal optimal smoothing formula with $h=1.06 \mathrm{~s} n^{-\frac{1}{5}}$, also $g$ is a normal density function. Similar to Vasicek-based test, we can prove the test based on $B_{K}$ is consistent.

\section{Simulation Study}

A simulation study is performed to analyze the efficiency of the proposed test statistics. The critical values are determined using Monte Carlo simulation with 10,000 replicates at the significance level 0.05 . Namely, for the test statistic like $B_{V}$, we have generated $n$ samples from the standard normal distribution and calculated its sample value $\left(B_{V, 1}\right)$. We did this work for 10,000 simulated random samples and calculated $\left(B_{V, 1}, \ldots, B_{V, 10,000}\right)$. Since large values of $B_{V}$ reject the null hypothesis, we specify the 9500th order statistic $\left[B_{V,(9500)}\right]$ as the critical value of $B_{V}$ (because $\alpha=0.05$ ).

Based on (10) different values of $m$ are given in Table 1. Table 2 gives the critical values of $B_{V}, B_{K}$ and the competitor tests for various sample sizes. As can be seen in Table 2, the critical values of $B_{V}$ and $B_{K}$ decrease as the sample size $n$ increase unlike other competitors. The reason for this is that in the proposed test large values of the test statistics reject the null hypothesis (in other competitor tests, small values reject the null hypothesis). So, the power of the tests ( $\mathrm{P}\left(\right.$ reject $H_{0} \mid H_{1}$ is True)) tends to one when $n$ increases and we expect this matter because the test is consistent. That is, the test statistic estimates $D_{B S}(f, g)$ which is zero under the null hypothesis. So, we expect that the estimator $B_{V}$ (and therefore critical values) tend to zero under the null hypothesis and as $n$ increases. 
Table 1: The values of $m$ for test $B_{V}$.

\begin{tabular}{cc}
\hline$n$ & $m$ \\
\hline $5-9$ & 2 \\
$10-19$ & 3 \\
$20-29$ & 5 \\
$30-49$ & 9 \\
$50-79$ & 15 \\
$80-100$ & 24 \\
\hline
\end{tabular}

Table 2: Critical values of $B_{V}$ and $B_{K}$ and other competitor tests.

\begin{tabular}{ccccccc}
\hline$n$ & $W$ & $A^{2}$ & $K_{n, m}$ & $K_{n, m}^{c}$ & $B_{V}$ & $B_{K}$ \\
\hline 5 & 0.775 & & 1.509 & 2.679 & 0.259 & 0.018 \\
6 & 0.792 & & 1.632 & 2.683 & 0.255 & 0.024 \\
7 & 0.809 & & 1.741 & 2.736 & 0.246 & 0.028 \\
8 & 0.823 & 0.667 & 1.815 & 2.790 & 0.237 & 0.030 \\
9 & 0.834 & 0.678 & 1.940 & 2.833 & 0.228 & 0.031 \\
10 & 0.845 & 0.686 & 2.038 & 3.020 & 0.194 & 0.031 \\
11 & 0.855 & 0.693 & 2.112 & 3.052 & 0.189 & 0.031 \\
12 & 0.861 & 0.699 & 2.212 & 3.091 & 0.183 & 0.032 \\
13 & 0.869 & 0.703 & 2.266 & 3.149 & 0.174 & 0.031 \\
14 & 0.875 & 0.707 & 2.334 & 3.178 & 0.170 & 0.030 \\
15 & 0.882 & 0.711 & 2.396 & 3.235 & 0.161 & 0.031 \\
20 & 0.904 & 0.722 & 2.707 & 3.403 & 0.135 & 0.029 \\
25 & 0.920 & 0.729 & 2.875 & 3.512 & 0.117 & 0.026 \\
30 & 0.930 & 0.733 & 2.996 & 3.606 & 0.118 & 0.024 \\
40 & 0.945 & 0.738 & 3.159 & 3.725 & 0.097 & 0.021 \\
50 & 0.954 & 0.741 & 3.318 & 3.801 & 0.102 & 0.019 \\
60 & 0.961 & 0.743 & 3.390 & 3.870 & 0.088 & 0.017 \\
70 & 0.965 & 0.745 & 3.450 & 3.887 & 0.080 & 0.015 \\
80 & 0.969 & 0.746 & 3.443 & 3.948 & 0.092 & 0.014 \\
90 & 0.972 & 0.747 & 3.515 & 3.978 & 0.084 & 0.013 \\
100 & 0.975 & 0.747 & 3.571 & 4.029 & 0.078 & 0.013 \\
\hline
\end{tabular}

In order to compute the powers of $B_{V}$ and $B_{K}$, a battery of Monte Carlo simulation was 
Table 3: Critical values of $A_{n, m}(m)$ test.

\begin{tabular}{ccccccccccc}
\hline & \multicolumn{1}{c}{$m$} & \multicolumn{1}{c}{$m$} & & & & & \\
\hline$n$ & 1 & 2 & 3 & 4 & 5 & 6 & 7 & 8 & 9 & 10 \\
\hline 5 & 3.198 & 3.309 & & & & & & & & \\
6 & 3.209 & 3.302 & 3.386 & & & & & & & \\
7 & 3.243 & 3.293 & 3.363 & & & & & & & \\
8 & 3.273 & 3.314 & 3.381 & 3.441 & & & & & & \\
9 & 3.302 & 3.333 & 3.385 & 3.441 & & & & & & \\
10 & 3.336 & 3.350 & 3.391 & 3.440 & 3.481 & & & & & \\
11 & 3.373 & 3.383 & 3.417 & 3.458 & 3.498 & & & & & \\
12 & 3.385 & 3.386 & 3.417 & 3.459 & 3.495 & 3.527 & & & & \\
13 & 3.429 & 3.421 & 3.442 & 3.477 & 3.516 & 3.547 & & & & \\
14 & 3.443 & 3.434 & 3.452 & 3.482 & 3.512 & 3.545 & 3.573 & & & \\
15 & 3.457 & 3.448 & 3.463 & 3.490 & 3.521 & 3.550 & 3.576 & & & \\
20 & 3.547 & 3.521 & 3.518 & 3.531 & 3.550 & 3.573 & 3.595 & 3.616 & 3.635 & 3.653 \\
25 & 3.621 & 3.598 & 3.586 & 3.588 & 3.599 & 3.615 & 3.634 & 3.651 & 3.666 & 3.681 \\
30 & 3.671 & 3.647 & 3.633 & 3.629 & 3.631 & 3.639 & 3.651 & 3.664 & 3.678 & 3.691 \\
40 & 3.748 & 3.724 & 3.708 & 3.700 & 3.695 & 3.696 & 3.699 & 3.705 & 3.713 & 3.722 \\
50 & 3.794 & 3.773 & 3.758 & 3.748 & 3.742 & 3.738 & 3.737 & 3.738 & 3.742 & 3.747 \\
60 & 3.831 & 3.812 & 3.796 & 3.785 & 3.777 & 3.771 & 3.768 & 3.766 & 3.767 & 3.769 \\
70 & 3.866 & 3.847 & 3.832 & 3.822 & 3.813 & 3.807 & 3.802 & 3.799 & 3.798 & 3.798 \\
80 & 3.892 & 3.872 & 3.858 & 3.847 & 3.839 & 3.832 & 3.828 & 3.824 & 3.822 & 3.821 \\
90 & 3.904 & 3.887 & 3.874 & 3.864 & 3.855 & 3.848 & 3.842 & 3.838 & 3.835 & 3.833 \\
100 & 3.922 & 3.906 & 3.893 & 3.882 & 3.873 & 3.865 & 3.859 & 3.855 & 3.850 & 3.848 \\
\hline
\end{tabular}

also applied. We regard the test statistics of normality discussed in Choi (2008) as the competitor tests. These tests are the Vasicek test $\left(K_{n, m}\right)$, the Anderson-Darling test $\left(A^{2}\right)$, the Shapiro-Wilk test $(W)$ (these three tests have the greatest power among the seven tests of Alizadeh and Arghami (2011a)) and the Choi test $\left(K_{n, m}^{c}\right)$. Moreover, the proposed test with the Alizadeh (2010) test $A_{m, n}$ was compared. To compare powers, we consider 7 alternatives used by Choi (2008) which are Uniform $(0,1)$, Exponential (1), Weibull $(1.2,2)$, Gamma $(1.5,2)$, Lognormal $(0,1)$, Chi-squared (2) and Inverse Gaussian $(1,1)$. For each alternative, we generated 10,000 samples of size 10, 20, 30, 50 and 100. For each sample the statistics $\left(A^{2}, W, K_{n, m}, K_{n, m}^{c}, A_{n, m}(m), B_{K}\right.$ and $\left.B_{V}\right)$ were evaluated and the power of the corresponding test was estimated by the frequency of the event "the statistic is in the critical region". 
Table 4: Estimated powers of the $B_{V}$ and the $B_{K}$ tests against the non-normal distribution at the significance level 0.05. (The best values are bolded)

\begin{tabular}{|c|c|c|c|c|c|c|c|c|}
\hline \multicolumn{9}{|c|}{ Test statistic } \\
\hline Distribution & Sample size & $A^{2}$ & $W$ & $K_{n, m}$ & $K_{n m}^{c}$ & $A_{m, n}(m)$ & $\overline{B_{V}}$ & $B_{K}$ \\
\hline \multirow{5}{*}{ Uniform $(0,1)$} & 10 & 0.084 & 0.087 & 0.174 & 0.153 & $0.152(5)$ & 0.105 & 0.015 \\
\hline & 20 & 0.169 & 0.198 & 0.432 & 0.443 & $0.451(10)$ & 0.357 & 0.028 \\
\hline & 30 & 0.300 & 0.388 & 0.649 & 0.704 & $0.734(15)$ & 0.604 & 0.186 \\
\hline & 50 & 0.582 & 0.755 & 0.928 & 0.969 & $0.974(25)$ & 0.915 & 0.662 \\
\hline & 100 & 0.948 & 0.997 & 1 & 1 & 1(45) & 1 & 0.989 \\
\hline \multirow{5}{*}{ Exponential(1) } & 10 & 0.406 & 0.438 & 0.423 & 0.455 & $0.457(4)$ & 0.494 & 0.354 \\
\hline & 20 & 0.771 & 0.832 & 0.847 & 0.854 & $0.859(9)$ & 0.882 & 0.774 \\
\hline & 30 & 0.934 & 0.967 & 0.970 & 0.970 & $0.979(13)$ & 0.982 & 0.934 \\
\hline & 50 & 0.996 & 1 & 0.999 & 0.999 & 1(25) & 1 & 0.998 \\
\hline & 100 & 1 & 1 & 1 & 1 & 1(1) & 1 & 1 \\
\hline \multirow{5}{*}{ Weibull(1.2,2) } & 10 & 0.283 & 0.310 & 0.276 & 0.302 & $0.324(4)$ & 0.337 & 0.253 \\
\hline & 20 & 0.584 & 0.659 & 0.645 & 0.665 & $0.692(9)$ & 0.882 & 0.607 \\
\hline & 30 & 0.791 & 0.873 & 0.849 & 0.879 & $0.899(13)$ & 0.907 & 0.806 \\
\hline & 50 & 0.961 & 0.989 & 0.986 & 0.986 & $0.986(22)$ & 0.990 & 0.979 \\
\hline & 100 & 1 & 1 & 1 & 0.992 & 1(1) & 1 & 1 \\
\hline \multirow{5}{*}{$\operatorname{Gamma}(1.5,2)$} & 10 & 0.285 & 0.310 & 0.266 & 0.295 & $0.326(4)$ & 0.331 & 0.272 \\
\hline & 20 & 0.589 & 0.661 & 0.625 & 0.644 & $0.687(8)$ & 0.698 & 0.607 \\
\hline & 30 & 0.795 & 0.870 & 0.829 & 0.855 & $0.893(13)$ & 0.895 & 0.818 \\
\hline & 50 & 0.964 & 0.987 & 0.979 & 0.981 & $0.993(23)$ & 0.987 & 0.976 \\
\hline & 100 & 1 & 1 & 1 & 0.986 & 1(1) & 1 & 1 \\
\hline \multirow{5}{*}{ Lognormal $(0,1)$} & 10 & 0.576 & 0.607 & 0.569 & 0.602 & $0.622(4)$ & 0.642 & 0.521 \\
\hline & 20 & 0.906 & 0.931 & 0.926 & 0.932 & $0.943(9)$ & 0.947 & 0.908 \\
\hline & 30 & 0.984 & 0.992 & 0.988 & 0.991 & $0.995(14)$ & 0.996 & 0.982 \\
\hline & 50 & 0.999 & 1 & 1 & 1 & 1(20) & 1 & 0.999 \\
\hline & 100 & 1 & 1 & 1 & 1 & 1(8) & 1 & 1 \\
\hline \multirow{5}{*}{ Chi-squared(2) } & 10 & 0.411 & 0.445 & 0.430 & 0.452 & $0.459(4)$ & 0.496 & 0.349 \\
\hline & 20 & 0.779 & 0.839 & 0.852 & 0.859 & $0.863(9)$ & 0.890 & 0.773 \\
\hline & 30 & 0.936 & 0.970 & 0.971 & 0.974 & $0.980(14)$ & 0.984 & 0.935 \\
\hline & 50 & 0.997 & 0.999 & 0.999 & 1 & 1(20) & 1 & 0.998 \\
\hline & 100 & 1 & 1 & 1 & 1 & 1(1) & 1 & 1 \\
\hline \multirow{5}{*}{ Inverse Gaussian(1,1) } & 10 & 0.515 & 0.542 & 0.554 & 0.529 & $0.536(4)$ & 0.574 & 0.466 \\
\hline & 20 & 0.877 & 0.907 & 0.894 & 0.900 & $0.921(10)$ & 0.925 & 0.880 \\
\hline & 30 & 0.973 & 0.986 & 0.978 & 0.983 & $0.990(14)$ & 0.990 & 0.977 \\
\hline & 50 & 1 & 1 & 1 & 1 & 1(21) & 1 & 1 \\
\hline & 100 & 1 & 1 & 1 & 1 & 1(35) & 1 & 1 \\
\hline
\end{tabular}


The goodness of fit test based on entropy involves choosing the best integer parameter $m$. Unfortunately, there are no choices out of the criterion of $m$, and in general it depends on the alternative. Vasicek (1976) selected the values of $m$, which maximize the powers of the test. It is shown by Alizadeh (2010) that, there is no $m$ being appropriate for all alternatives. The values of $m$ that attain maximum powers are also suggested and presented in Table 1 for the $B_{V}$ test. Table 4 shows the estimated power of the tests $B_{V}$ and $B_{K}$ and those of the competing tests, at the significance level $\alpha=0.05$ based on 10,000 iterations with sample sizes 10, 20, 30, 50 and 100.

According to Table 4, for a symmetric alternative means uniform distribution, the greatest power is related to $K_{n, m}$ test for $n=10$, and to $A_{m, n}$ test for $n=20, n=30$ and $n=50$. However, for the asymmetric alternatives, the power of the $B_{V}$ test is better than the other tests for all alternatives and for all sample sizes (except Gamma when $\mathrm{n}=50$ ). In addition, the $B_{V}$ test, has the advantage of owning a fixed $m$ in comparison with $A_{m, n}$ test. So, we can suggest the $B_{V}$ test as a test of fit for normality for asymmetric alternatives.

Concerning $B_{K}$, it can be understood that its powers are weaker in comparison with the other tests (except $A^{2}$ for large sample sizes in most of the times). But $B_{K}$, just has the advantage of being independent on fixed $m$.

\section{Conclusion}

In the present paper, a goodness of fit test for normality based on Balakrishnan-Sanghvi divergence was introduced. To construct the test statistic, two methods were investigated. The first method was similar to Vasicek's method for estimating the Shannon entropy and the second one, was based on kernel density function. By a simulation study, the powers of the proposed tests were computed under several alternatives and different sample sizes. It is shown that, the $B_{V}$ test, compares favorably with the leading competitors for every sample size especially for the asymmetric alternatives.

\section{References}

Ahmad, I. A. and Lin, P. E. (1976), A nonparametric estimation of the entropy of the absolutely continuous distribution. IEEE Transactions on Information Theory, 22, 327-375. 
Alizadeh Noughabi, H. (2010), A new estimator of entropy and its application in testing normality. Journal of Statistical Computation and Simulation, 86, 1151-1162.

Alizadeh Noughabi, H. and, Arghami, N. R. (2011a), Monte Carlo comparison of seven normality tests. Journal of Statistical Computation and Simulation, 8, 965-972.

Alizadeh Noughabi, H. and Arghami, N. R. (2011b), Testing exponentiality based on characterizations of the exponential distribution. Journal of Statistical Computation and Simulation, 81, 1641-1651.

Anderson, T. W. and Darling, D. A. (1954), A test of goodness of fit. Journal of the American Statistical Association, 49, 765-769.

Arizono, I. and Ohta, H. (1989), A test for normality based on Kullback Leibler information. The American Statistician, 43, 20-23.

Balakrishnan, V. and Sanghvi, L. D. (1968), Distance between populations on the basis of attribute. Biometrics, 24, 859-865.

Choi, B. (2008), Improvement of goodness of fit test for normal distribution. J. Stat. Computat. Simul., 78, 781-788.

Corea, J. C. (1995), A new estimator of entropy. Communications in Statistics - Theory and Methods, 24, 2439-2449.

Cramer, H. (1928), On the composition of elementary errors. Scandinavian Actuarial Journal, 1, 13-74.

Dudewicz, E. S. and Van der Meulen, E. C. (1981), Entropy-based tests of uniformity. Journal of the American Statistical Association,76, 967-974.

Ebrahimi, N., Habibullah, M. and Soofi, E. S. (1992), Testing exponentiality based on Kullback-Leibler information. Journal of the Royal Statistical Society. Series B (Methodological), 54, 739-748.

Ebrahimi, N., Pflughoeft, K. and Soofi, E.S. (1994). Two measures of sample entropy. Statistics \& Probability Letters, 20, 225-234.

Esteban, M. D., Castellanos, M. E., Morales, D. and Vajda I. (2001). Monte Carlo comparison of four normality tests using different entropy estimates. Communications in Statistics - Simulation and Computation, 30 761-785. 
Finkelstein, J. and Schafer, R. E. (1971), Imported goodness of fit tests. Biometrika, 58, 641-645.

Jarque, C. M. and Bera, A. K. (1987), A test normality of observations and regression residuals. International Statistical Review / Revue Internationale de Statistique, 55, 163172.

Joe, H. (1989), Estimation of entropy and other functionals of a multivariate density. Annals of the Institute of Statistical Mathematics, 41, 683-697.

Kolmogorov, A. N. (1933), Sulla determinazione empirica di una legga di distribuzione. Giornale dell Istituto Italiano degli Attuari, 4, 83-91.

Kuiper, N. H. (1960), Tests concerning random points on a circle. Indagationes Mathematicae (Proceedings), 63, 38-47.

Park, S. (1999), A goodness-of-fit test for normality based on the sample entropy of order statistics. Statistics \& Probability Letters, 44(4), 359-363.

Pearson, K. (1900), On the criterion that a given system of deviations from the probable in the case of a correlated system of variables is such that it can be reasonably supposed to have arisen from random sampling. The London, Edinburgh, and Dublin Philosophical Magazine and Journal of Science, 50, 157-175.

Smirnov, N. (1948), Table for estimating the goodness of fit of empirical distributions. The Annals of Mathematical Statistics, 19(2), 279-281.

Song, K. S. (2002), Goodness-of-fit tests based on KullbackLeibler discrimination information. IEEE Transactions on Information Theory, 48(5), 1103-17.

Shannon, C. E. (1948), Mathematical theory of communications. Bell System Technical Journal, 27, 379-423.

Shapiro, S. S. and Wilk, M. B. (1965), An analysis of variance test for normality (Complete Sample). Biometrika, 52, 591-611.

Vasicek, O. (1976), A Test for normality based on sample entropy. Journal of the Royal Statistical Society. Series B (Methodological), 38, 730-737.

Van Es, B. (1992), Estimating Functionals Related to a Density by a Class of Statistics Based on Spacings. Scandinavian Journal of Statistics, 19, 61-72. 
Von Mises, R. E. (1928), Wahrscheinlichkeit, Statistik und Wahrheit. Julius Springer.

Von Mises, R. (1931), Wahrscheinlichkeitsrechnung und ihre Anwendung in der statistik und theoretischon physik. Deuticje, Leipzing and vienna.

Wang, X., Liu, Y. and Han, B. (2018), Goodness-of-fit tests based on Bernstein distribution estimator. Journal of Nonparametric Statistics, Submitted.

Watson, G. S. (1961), Goodness of fit tests on a circle. Biometrika, 48, 109-114.

Wieczorkowski, P. and Grzegorzewsky, P. (1999), Entropy estimators improvements and comparisons. Communications in Statistics - Simulation and Computation, 28, 541567.

Yousefzadeh, F. and Arghami, N. R. (2008), Testing exponentiality based on type II censored data and a New cdf estimator. Communications in Statistics - Simulation and Computation, 37, 1479-1499. 